\title{
Balancing act: The relentless tyranny of monitoring and evaluating body image concerns
}

\author{
KEITH TUFFIN, JENNIFER HAMID AND DENISE BLAKE
}

\begin{abstract}
This article outlines an exploratory grounded theory of women's experiences regarding body image. Eleven women, aged 18 to 25, participated in semi-structured interviews. The study explored a range of concerns, focusing on weight, size and appearance; the participants discussed how these concerns affected them, and what contributed to their concerns. The preliminary grounded theory suggests that all the women, to various degrees, monitor, evaluate and/or alter their bodies in specific ways to manage social relationships and maximise positive feelings about themselves, while minimising negative ones. The theory that emerged from this study is process oriented and suggests that for most of the women being happy with their bodies, or not feeling unhappy with them, is only a transitory part of an ongoing cyclical process of monitoring and evaluation. This theory gives rise to research possibilities which are discussed.
\end{abstract}

\section{Key words}

grounded theory, women, body image, monitor, evaluate

\section{Introduction}

The prevalence of women's body image concerns represents normative discontent (Rodin, Silberstein, \& Striegel-Moore, 1984), involving widespread cultural preoccupation with weight, physical size and body dissatisfaction. Notions of body image are complex and can encompass perceptual, emotional and behavioural aspects of experience. More recently body image has been taken to represent body shape, size and weight (Tiggemann, 2004). Normative discontent is pervasive in Western society (Matthiasdottir, Jonsson, \& Kristjansson, 2010), and extends beyond those with diagnosable eating disorders (Streeter, Milhausen, \& Buchholz, 2012). It is now normal for women to feel dissatisfaction with their bodies, to diet, and to worry about their weight and appearance (Jackson \& Lyons, 2012; Markula, Burns, \& Riley, 2008; TantleffDunn, Barnes, \& Larose, 2011). Research has documented overwhelming numbers of women who feel too fat and therefore engage in repeated efforts to lose weight by dieting and exercising (see for example Hausenblas \& Fallon, 2006; Leong, Madden, Gray, \& Horwath, 2012).

Negative body image is a predictor of depression, obesity and disordered eating and the increasing numbers experiencing distress around body image is disturbing (Hausenblas \& Fallon, 2006). According to one American study of 2279, 29.3\% of 10-14-year-old girls were trying to lose weight, while $10.5 \%$ passed the threshold for disordered eating (McVey, Tweed, \& Blackmore, 2004). Longitudinal research by Neumark-Sztainer, Wall, Larson, Eisenbergy and Loth (2011) found increases in extreme dieting and disordered eating for adolescent girls and, worryingly, these behaviours remained constant or increased into young adulthood. Advocating for early intervention, the authors argue that extreme weight control in middle adolescence increases the risk of harmful behaviours 10 years later. Although eating disorders and normative discontent are more common among younger women, they are also found in older women (Goodheart, Clopton, \& Robert-McComb, 2012; Tantleff-Dunn, et al., 2011).

Weight and body image are important determinants of social and psychological wellbeing (Hesse-Biber, 1991). Constituted within Western cultural standards of thinness, women learn 
to associate self-worth with appearance (Davis \& Cowles, 1991; Featherston, 2010). Being fit, toned and slender equates to success and social acceptance (Grogan, 2008). Social discourses represent women as desirable if they are less visible and occupy a minimum amount of physical space; bodies need to be small and exhibit minimal evidence of female secondary sexual characteristics (Bordo, 2003; Chernin, 1981). Obese women have a poorer body image, independent of body-altering practices, such as exercise (Streeter, et al., 2012). Yet while dieting holds the promise of alleviating negative body image, sustained reduction in body weight is mostly unattainable, with effects typically being short-lived and psychologically draining. There are increased health risks, even mortality, with repeated weight loss and gain over long periods of time (Burns \& Gavey, 2004; Hill, 2004). While food restricting practices are usually unable to permanently reduce body size, feelings of a 'self' as fat or unacceptable construct and reproduce the insecure body and ongoing experiences of normative discontent (Rodin, et al., 1984; Streeter, et al., 2012).

The history of women altering their bodies in the pursuit of contemporary beauty ideals has long been documented (Bordo, 1993, 2003; Burns \& Gavey, 2008; Featherston, 2010; HesseBiber, 1991; Jeffreys, 2005; Rodin, et al., 1984). These practices, such as wearing tight corsets in Victorian times and the Chinese custom of foot binding, resulted in medical problems including the displacement of internal organs, difficulties in walking and severe pain. Today practices of restricted eating, extreme or fad diets and cosmetic surgery all carry serious health risks, but are commonly enacted and accepted as normal (Bordo, 2003; Grogan, 2008; Jackson \& Lyons, 2012). Expectations to achieve the desired beauty ideals operate through internally imposed restrictions, drawn from cultural norms and feminine stereotypes, rather than externally enforced restrictions. However, the subtlety of the 'psychological corset' is no less damaging than the more obvious physical constraints seen throughout history (Bartky, 1988; Bordo, 1993, 2003). The psychological corset that is a requirement of the thin ideal involves stringent self-monitoring and control.

The ideals of a virtuous body that is desirable (Crawford, 2006; Donaghue \& Clemitshaw, 2012) means that thinness symbolises self-discipline, personal order and assertiveness (Bordo, 2003; Grogan, 2008). Women take up self-disciplining practices, such as diet and exercise, as a form of self-transformation, according to Heyes (2007). Here, the process of normalisation, and the need to meet dominant feminine ideals, both constrains and enables experience. Women are constrained to comply with body image ideals that simultaneously enable them to be and act in particular ways. This inseparable process is produced through the body and represents social beliefs that proscribe women's bodies as a representation of their inner traits.

The inability to reach the thinness ideal can be disempowering while overweight women are negatively stigmatised as unattractive and lazy. Cultural stereotypes represent the obese body as sloppy, dirty and stupid (Grogan, 2008; Markula, et al., 2008). The problematisation of woman's bodies as being in constant need of alteration positions them as 'patients' needing 'treatment'. Women are produced and reproduced within socio-political climates such as the neoliberal pursuit of economic growth and market driven economies. One of the arguments here is that the partnership of androcentric cultural ideology and corporate/capital interests (diet, beauty, cosmetic and health industries) controls women's bodies through socio-cultural pressures that position women as ideally thin (Bordo, 2003; Hesse-Biber, 1991). The economic rationale for the thin ideal is clear when the lucrative weight control and cosmetics industries are considered. Drawing on Foucault, Bartky (1988) contends that such disciplinary practices need to be understood in the context of 'patriarchal domination' (p. 64). She argues that although previous constraints upon women, such as requirements to be chaste and modest, or to restrict themselves and their activity to the home, are no longer in place, the modernisation 
and diffusion of patriarchal power has shifted constraints from external regulation (such as the church and the traditional nuclear family) to internal regulation, or more specifically, to constraints centred on body image. In a sense women become self-policing states; they internalise the social rules and regulations of the feminine ideal. They seek control over themselves, and obviate the need for externally imposed controls. This constraint to meet culturally prescribed ideals while being constructed as objects of the 'male gaze' means women begin habitual and relentless self-evaluation and monitoring of their external appearance (Tiggemann, 2004).

It has long been found that body image concerns are normative within Western and European cultures; there is more body image preoccupation here than within other ethnic group settings. However, dominant Western ideals permeate and influence other cultures. For example, Sabik, Cole and Ward (2010) found that African American women with affiliations outside of their ethnic orientation had a greater drive for thinness than those lower in other-group orientations. The media is implicated in the promulgation of the thinness ideal. Consumers are bombarded with messages to diet, lose weight, tone up and trim down, while simultaneously given messages to buy, prepare and consume the right kinds of products and food. While there is difference between idealised beauty as portrayed in the media and the shape, size and weight of 'real' women, it makes sense that women experience their bodies negatively (Tiggemann, 2004). As far back as 1993, Bordo suggested media promotion of the slender body contributes to risky behaviours such as extreme dieting and over exercising with the simple message being to avoid fat and anything that 'jiggles'. Jackson and Lyons (2012) discuss how women attempt to mould themselves to the desired norms by taking up body-altering practices that aim for the 'perfect' body and feminine ideals to fit the 'male gaze'. Trapped within a cycle of compulsive exercise and food management, many women measure, monitor and manage their body weight (Burns \& Gavey, 2004).

Normative discontent realises body altering practices as a culturally accepted norm, and restricted food practices, including fat and calorie counting, are now a 'lifestyle choice' constituted within the thinness ideal (Bordo, 2003; Tantleff-Dunn, et al., 2011), that necessitates constant self-monitoring and evaluation. In Western cultures powerful forces ensure the majority of women feel bad about their bodies, and as such these social power relations require ongoing attention.

\section{Research orientation}

The quantifiable aspects of women's body image dominate the psychological literature. Although this produces particular knowledge, the current study addresses how women experience their bodies and the ways in which monitoring and evaluation are expressed. To do this the second author conducted semi-structured interviews. As argued by Grogan (2008), interviews provide women with the space to have a voice to express their lived experience. Research grounded in what women say enables new possibilities for exploring women's experiences. Accordingly, this study involved a 'grounded theory' approach (Strauss, 1987; Strauss \& Corbin, 1990) to build a theory of women's perspectives and experiences of their body concerns in a method systematically related to their understandings. This research orientation provided an approach consistent with our research aims, enabling the integration of what we wanted to investigate (women's experiences) and how we wanted to investigate that within an exploratory, contextual and relational framework.

Our epistemological allegiance values multiple, relativistic context bound 'truths', 'realities', and 'ways of knowing'. This epistemic plurality underpins our commitment to the welfare of women and views the knower as inseparable from the known. Here, the research relationship is understood as interactive and inseparable; we acknowledge the subjectivity inherent 
in interpersonal interaction (such as research) and value the importance of reflexively producing explicit formative frameworks. We reflexively approach this research respecting the lived experience of the women who participated, and the importance of giving voice.

\section{Research process}

A grounded theory is generated inductively from the study of the phenomenon that the theory aims to represent (Strauss \& Corbin, 1990). In other words, theory is generated by the data, and is grounded in the data. Grounded theory is concerned with the development of theory, and as such is not a specific method. Rather, it is a style of qualitative analysis that includes its own distinct methods and techniques, such as theoretical sampling and the use of specific coding procedures, as well as methodological guidelines whereby there is constant comparison and open coding of data (Henwood \& Pidgeon, 2006; Strauss, 1987; Strauss \& Corbin, 1990). Strauss (1987) stresses that suggested methods in grounded theory be regarded as guidelines only, to be adapted to the specific research setting. Accordingly, grounded methods should encourage creativity and insight; two vitally important processes to developing a sensitive interpretation of data (Henwood \& Pidgeon, 2006).

The emphasis within a grounded theory approach is the generation of theoretical concepts, rather than verification of concepts (Henwood \& Pidgeon, 2006). However further into the theory development, the grounded theorist combines verification with generation, searching for both confirming and disconfirming examples of emerging categories in order to increase conceptual complexity (and therefore theoretical relevance) of the categories, and eventually of the network of categories that is the emerging theory (Henwood \& Pidgeon, 2006; Strauss \& Corbin, 1990). The integrity of a grounded theory is based on how well the theory is grounded in the data, how well it fits participants' experience, and the degree to which it seems believable and plausible to those who read it.

\section{Participants}

Eleven women aged between 18 and 25 participated in the study. While this research values data saturation rather than sample size, participant numbers reflect a qualitative epistemology that argues that new themes do not emerge after the first twelve interviews (Baker \& Edwards, 2012; Guest, Bunce, \& Johnson, 2006). The age group was chosen on the basis that most bodily dissatisfaction is likely to occur between late teens and early twenties (Neumark-Sztainer, et al., 2011; Streeter, et al., 2012). All of the women identified as Pākehā or European, representing a convenience sample of university students. Ethnicity has implications for body dissatisfaction - for example more European American women attach self-worth to appearance than other ethnicities, and increased mental health symptoms are associated with eating disorders (Grabe \& Hyde, 2006). Notices were put up on a university campus asking for women volunteers to assist with an interview-based study on aspects of body image. The women did not identify as having eating disorders, and dress sizes ranged from 8 to 16, representing a range of body shapes and sizes.

\section{Procedure}

Participants were interviewed separately, with interviews lasting between 30-60 minutes. Interviews were organised around questions and prompts that asked participants what they thought, how they felt, and what they did in relation to their body image. Questions focussed on weight, shape, size and appearance, how these concerns affected participants personally and socially; and finally, what they perceived as having contributed to their concerns. 
The topics and experiences that were important to the women guided the interviews and the direction of subsequent questions (Henwood \& Pidgeon, 2006; Strauss, 1987). Any theoretically relevant issues mentioned in the early interviews were inquired about in the subsequent interviews, with encouragement for the women to elaborate. This ongoing process meant that any new themes were followed up in the next interviews. In this way, the interview 'structure' developed as the research progressed. All interviews were transcribed with participants having chosen a pseudonym to protect their confidentiality, while ethical approval was granted by the Massey University Ethics Committee.

\section{A grounded theory of women's body image}

The importance of managing body image was evident, with participants expressing concern about the image they presented to others. The intensity of how the women managed themselves depended on how they experienced their bodies: the less happy they were with their bodies at any particular time, the more intensely they 'checked' themselves:

Yeah, I am very self-conscious to the extent that I always check to make sure I look okay before I leave, but it's not, it's only, yeah it's mainly during the periods that I do think I'm not looking very good. But I guess I wouldn't be self-conscious if I think I look good, then I don't really care. (Alison)

To manage feeling 'self-conscious' and 'not looking good' around others, Alison monitors and evaluates herself before she leaves the house. Monitoring can be understood as a process of watching or 'checking' the self. Evaluating is the concurrent process of making judgements about the results of that monitoring. These processes are intertwined, ongoing and cyclical. When Alison experiences a positive self-image, she 'doesn't really care' how she appears to others, and does not feel the need to 'always check'.

The intensity of monitoring ranged from some being vaguely aware (low intensity), through to vigilance (high intensity). High intensity monitors evaluated themselves more often and more harshly, felt more self-conscious, and were much stricter on themselves than low intensity monitors. The process of monitoring and evaluation varied across situations; the worse a woman felt in a social situation the more intensely she monitored herself:

I find that if I'm in a situation where there's a lot of people and when the emphasis is on looking good, like at a party, or maybe at a pub or, you know when you go out with a lot of people, and then I notice myself looking at myself in that way. You know, the old spectator again, and then I feel more uncomfortable. (Lauren)

Cultural and social pressure on body image is implicated. For Lauren the pressure to manage her body image causes critical self-analysis, and the 'old spectator' begins monitoring and evaluating again. This process is 'uncomfortable' on the one hand, but necessary and familiar to manage body image concerns. The more 'uncomfortable' Laruen feels, the more she 'looks at herself', indicating a cyclical process. The public body produces vigilance, especially when the risk involves more intimate exposure:

I really hate going to swimming pools and stuff, because I've got to wear togs and, you know, I feel really self-conscious about that. And I hate going to the beach in summer and having to lie there in my togs on the beach. Yeah, so [it's] the situations where you've got to show your body. You know, emphasis is sort of placed on your body shape. (Joanne)

Like if I go to the local pool and there's males around I sort of ... I don't even go to the local pool because there's usually males there, It's just a thing ... Cos they make you wear swimming togs there. (Tracey)

Joanne reports a strong dislike of situations where her body is publicly displayed. These situations emphasise body shape and the centrality of this increases her level of self-consciousness. Similarly, Tracey dislikes the local pool because of the male gaze, and as a way to cope she enacts avoidance strategies. Avoiding situations that provoke critical self-evaluation prevents 
women from experiencing negative and often distressing emotional effects. Situations are avoided entirely because of self-monitoring and evaluation processes that are not 'comfortable':

I do [avoid situations because I feel self-conscious] to the extent of situations with strangers ... but not with friends ... it comes back down to whether I feel comfortable in a situation, like I do with friends but not so much with strangers. (Jane)

For Jane, it is much easier to deal with friends when 'self-conscious' rather than strangers. The public display of bodies heightens the level of scrutiny of the body with negative effects. The history of negative male judgements tightens the 'psychological corset', which is important because it becomes internalised and continues to have consequences for the experience of self:

When I was young I can remember being teased as a little girl about being fat, and, as much as I can think of what happened, I think it really has. You know, just comments that people have passed, or, you know, boys being cruel in intermediate and stuff like that. I think that I would have to say that would definitely have an effect on a girl, especially by a guy, a dire effect. (Sharon)

Sharon talks about the cruel and ongoing effects of negative judgements. Here the male gaze matters to Sharon's perception of herself, with the effects being 'dire'. What is more, the experience of feeling evaluated by others can occur even if no comment is made. The mere presence of men is sufficient to make some women feel uncomfortable and influence their behaviour:

\footnotetext{
When there was a group of guys I'd walk across the street rather than walk past them because I felt my body was being evaluated. I just felt, you know, really uncomfortable and just wanted to hide under my coat or whatever or crawl into a hole! Yeah, crossing the street and also covering my body with baggy clothes and especially if I feel uncomfortable about a certain part of my body. It's like before my period if my breasts are poking out or, you know, then I'd sort of hunch my shoulders over or cross my arms or something like that. (Lauren)
}

I just withdraw and wear baggy clothes and look disgusting so nobody ever really pays any attention to what I'm wearing or how I feel, sort of like cover yourself up. (Lee)

While being evaluated, women can experience negative feelings (such as discomfort or embarrassment). 'Crawling into a hole' and 'hunching' the body while feeling judged represent effects of exposure and bodily restrictions that signifies a body that has to be less visible and small. To look 'disgusting' and to 'cover up' can be understood as forms of protection and resistance to the cultural pressure to enact particular feminine ideals, such as having a slender body. Here, the body is hidden. Monitoring and evaluating the self as protection from others' judgements has consequences for relationships. Body image concerns about 'bulging cheeks' means Lee does not 'smile because her face is too fat'; this evaluation restricts Lee from showing warmth when encountering kindness:

If people do something nice for me I don't smile at them because I think my face is too fat to smile. I think my cheeks bulge out. And so, like, if someone does something nice to me, like the guy at the petrol station, I just get all embarrassed and go away, rather than being nice, because I think it's probably making him sick. (Lee)

Becoming less visible is better than making others 'sick' or having feelings of 'embarrassment', but hinders engagement in social relationships. The monitoring and evaluation process necessarily produces a constant vigilance about self-image. This vigilance is constituted as organic; it is 'ingrown':

I mean I'm always, constantly evaluating myself...it's an ingrown thing. (Lauren)

Representing a metaphor of a painful 'ingrown' bodily process, constant monitoring can be seen as agonising and injurious. The internalisation of monitoring, we suggest, may be fashioned by the psychological corset that is relentless and too tight. 
To manage the pain, the monitoring and evaluating of 'self' required a complex mental juggling act to keep account of four separate 'balances', namely weight, food intake (both amount and content), exercise and health. Some participants experienced negative feelings if these levels became unbalanced (to a self-specified degree), and here they would try to engender more positive feelings by 're-balancing the books':

If I do a lot of exercising, then I can eat what I want - there definitely is a balance. (Maria)

If I feel that I've overeaten I tend to feel bad or guilty and then I say, 'well as long as I go and do exercise, then it's going to be okay', like it cancels itself out. 'You can eat this if you do this later on' and it's sort of like living to rules and, you know, keeping to everyday rules. Yeah, I suppose it's like a balance really, that if I eat this much then I've got to do that much exercise, or if I eat that much then I'm only allowed to eat that much tomorrow or for dinner, or... So it's almost like I've got a little pair of scales in my head, you know. (Lauren)

The ongoing negotiation and 'balancing' feels like internal 'scales' that are a permanent experience in Lauren's 'head'; it involves a set of rules to be obeyed 'everyday'. To avoid experiencing insecurity about their bodies, the need to 'balance' exercise and food was important, and all participants claimed to monitor and practise particular 'balancing' techniques at some time.

Alongside the need to 'balance the books', monitoring and evaluating occurred in two distinct ways: in relation to others, and in relation to the self. Monitoring in relation to others involved the management of external influences on women's thoughts, feelings and behaviour. These influences included Western cultural ideals as represented through family and peers, the media, and the fashion and diet industries. The effects of these influences meant women constantly checked and rechecked that their body image was representing the required feminine ideals.

A woman's position on the monitoring continuum was also directly related to how she felt about herself, and what she did, thought and felt about her body. Positive body image and balancing practices were associated with less monitoring, and less positive body image was linked with higher levels of monitoring. Each position required different balancing practices, depending on how a woman felt about herself. Two of the women reported reasonably low level monitoring and were generally happy with their bodies; they did not experience many negative feelings in relation to their bodies at all. Six of the women reported that they vary in their intensity of monitoring from mid intensity (watching, or watching closely), to high intensity (vigilance). Sometimes these women felt happy with their bodies, but not always, and they also experienced negative feelings about their body image. The remaining three women reported that they monitor themselves at a high intensity; that is, they were always vigilant, very critical of themselves and often felt negative about their bodies. The constraints of needing to 'balance the books' represent both the negative effects of social compliance and the positive effects of social transformation.

\section{Discussion}

This research into body image is generally consistent with and supports the existing literature in this area. Our theoretical framework focuses on the process and cyclical nature of the ways women attempt to regulate and/or alter their bodies in order to maximise positive feelings about themselves, and avoid normative discontent. Self-consciousness, monitoring and evaluation were accentuated in social situations where it was important to look good when in the company of others. The findings of this study suggest that maximising positive feelings is a major motivating factor for women's attempts to monitor and manage their bodies, and that this is strongly linked with social relationships. In contemporary Western society those who aim for or achieve the feminine ideals are rewarded and receive social, personal and interper- 
sonal success. For example, women can be complimented for achieving or maintaining a body size deemed more physically desirable within heterosexual relationships; they can also experience a greater sense of internal esteem whereby "“an outer self" [is] brought into line with an inner truth' (Heyes, 2007, p. 21). The pervasive disapproval and negative stereotyping of obesity encourages women to avoid it, or any image of it, at all costs. Together, these two processes provide a powerful incentive for women to regulate their body size and shape through 'balancing the scales'. Women are enabled and constrained in the process of normalisation towards the feminine ideal (Heyes, 2007).

The continually occurring processes of monitoring and evaluation, while balancing weight, food intake (both amount and content) and exercise were the main components in the women's control or management of themselves. These processes are so commonplace and so integrated into the women's lives that it is almost like background noise. Women monitor and evaluate themselves daily - going to social events, swimming, walking down the street or buying petrol. A woman who worries about her appearance, her weight, her eating or anything to do with how she appears to others has become, in Bartky's (1988) words, 'a self-policing subject, a selfcommitted to a relentless self-surveillance' (p. 81). Lauren exemplifies the relentless nature of the cycle of monitoring and evaluating when she talks about continually checking her body, stating: 'It's like being on duty'.

In the present study, the women who were low intensity monitors - and reported that they were almost always happy with their bodies - were both a size 10; while those who ranged from mid intensity to high intensity monitoring were of various sizes (12-16). Interestingly, the high intensity monitors - the women who are most unhappy about their bodies - were sizes 8 to 12. This replicates previous research (see for example Markula, et al., 2008), suggesting that women's feelings and behaviours about their bodies are closely linked to their perceptions of weight and size. The women who were happy with their bodies perceived themselves as being acceptable, while the other women, to varying degrees, perceived themselves as not always acceptable.

Among our participants the differential effects of being happy or not with their bodies were substantial. The women who were generally happy with their bodies very rarely talked to themselves harshly, negatively or critically, while those who were unhappy with their bodies were often harsh, critical, and talk negatively to themselves. For all except two, enjoyment of life was diminished quite markedly - as in the case, to take but one example, of the participant who believed her face was too fat to smile and thus avoided certain social interactions and the expression of gratitude. These findings suggest that women who are unhappy with their weight or shape spend much more time and energy (both physical and emotional) on monitoring, management and balancing their weight/shape than those who are happy. Potentially this can be a negative and psychologically destructive process.

The theory developed in the present study is also consistent with the conceptualisation of 'body image disturbance/eating disordered behaviour' as a continuum (Rodin, et al., 1984). The women represented a range of feelings about their bodies, and a range of eating behaviours. However, deciding which of these feelings and behaviours are 'normal' or 'abnormal' is not possible unless we impose an arbitrary division based on a value judgement. And what is most important is that a division is not useful, except when identification would protect against potential harm.

The sample used in the present study was necessarily small, and restricted to a particular, homogeneous group of women. This allowed for in-depth interviews, and important generative analysis. Grounded theory values abstract concepts that can be applied to wider populations (Henwood \& Pidgeon, 2006), where the particulars of a grounded theory can be easily 
modifiable to incorporate new information. For instance, if future research should find that a particular group of women do not ever feel self-conscious about their bodies, the theory could easily be modified and expanded to include a conceptualisation of self-consciousness that varied along the dimension 'always - never'. This new dimension could then be explored to understand what causes and precedes self-consciousness. Also, grounded theorists emphasise theory that is always growing, modified and added to, and the present interpretation is no exception (Henwood \& Pidgeon, 2006; Strauss \& Corbin, 1990). This research represents the early stages of conception, and could be further developed with alternative populations, such as women of different ages, backgrounds, ethnicities, education and life perspectives.

The implications of viewing body image disturbance/eating disordered behaviour as a continuum and thus for seeing body image concerns as a public health issue are important. It would be useful to investigate the differences between women who are happy with their body image and those who are not. Once these differences are understood, more effective interventions such as education or therapy could be employed. Interventions could focus on assisting women to feel more positive about their bodies and be mindful of cultural pressures to attain contemporary feminine ideals of being thin. Other outcomes could be to challenge normative discontent, criticism, and negative feelings that arise from self-conscious and negative evaluation, as well as redirecting time and energy (physical, emotional and psychological) away from harmful body altering practices. This could enable the possibility for more positive psychological spaces to encourage women to be kinder to themselves, more confident and less restricted.

\section{Conclusion}

This study has provided a dynamic examination of the psychology of normative discontent and body dissatisfaction. Our analysis suggests a number of factors such as beliefs, interpretations and perceptions play a significant role in overall body image. These factors are important to how the women feel and how they act in relation to their bodies. Indeed, their perceptions of their own weight and size affect not only how they feel about their bodies, but also how they perceive others are evaluating them, thereby illustrating the way in which weight and body image are inextricably linked to social and psychological wellbeing.

The model developed in the present study is process oriented and highlights the continued importance of existing theoretical perspectives. Our examination of the symbolism of the psychological corset has enabled a better understanding of the lived experience of the tyranny of self-policing and the ways women remain continually duty bound and vigilant. The theoretical framework signifies the dynamic critical processes of monitoring and evaluation. These cyclical processes involve constant self-examination and judgement that produce particular coping strategies - all of which aim to preserve some sense of homeostatic balance. The theory suggests that for most of the women, being positive about their bodies is only a transitory part of an ongoing cyclical process of monitoring and regulating their bodies. This view of women's body image concerns as a process differs significantly from traditionally static models which tend to gloss over the process and the variability contained within the complex issue of body image concerns. In some sense, the women in the present study had relatively stable feelings towards their bodies. However, interwoven with this is the processual and cyclical nature of how the women relate to their bodies, involving continual monitoring, evaluation and balancing of the ledger.

KEITH TUFFIN is an Associate Professor in the School of Psychology, Massey University, Wellington, New Zealand. He teaches critical social psychology and discursive psychology. Current research interests include the language of racism, political discourse and constructionist approaches to emotion, culture and identity. 
JENNIFER HAMID was formerly a graduate research student in the School of Psychology at Massey University and has completed a Masters degree.

DENISE BLAKE is a lecturer in the School of Psychology at Massey University, Wellington. Her research interests include issues around social justice, adoption, addiction, and gender.

\section{References}

Baker, S. E., \& Edwards, R. (2012). National Centre for Research Methods review paper: How many qualitative interviews is enough? Expert voices and early career reflections on sampling and cases in qualitative research. University of Southampton, Southampton, England: National Centre for Research Methods.

Bartky, S. L. (1988). Foucault, feminism, and the modernization of patriarchal power. In I. Diamond \& L. Quimby (Eds.), Feminism and Foucault: Reflections on resistance (pp. 61-81). Boston, MA: Northeastern University Press.

Bordo, S. (1993). Unbearable weight: Feminism, Western culture and the body. California, CA: University of Calfornia Press.

Bordo, S. (2003). Unbearable weight: Feminism, Western culture, and the body (10th anniversary ed.). Berkeley, CA: University of California Press.

Burns, M., \& Gavey, N. (2004). 'Healthy weight' at what cost? 'Bulimia' and a discourse of weight control. Journal of Health Psychology, 9(4), 549-565. doi: 10.1177/1359105304044039

Burns, M., \& Gavey, N. (2008). Dis/orders of weight control: Bulimic and/or 'healthy weight' practices. In S. Riley, M. Burns, H. Frith, S. Wiggins \& P. Markula (Eds.), Critical bodies: Representations, identities and practices of weight and body management (pp. 139-154). London, England: Palgrave Macmillan.

Chernin, K. (1981). The obsession: Reflections on the tyranny of slenderness. New York, NY: Harper \& Row.

Davis, C., \& Cowles, M. (1991). Body image and exercise: A study of relationships and comparisons between physically active men and women. Sex Roles, 25(1-2), 33-44. doi: 10.1007/bf00289315

Featherston, M. (2010). Body, image and affect in consumer culture. Body \& Society, 16(1), 193-221. doi: $10.1177 / 1357034 X 09354357$

Goodheart, K. L., Clopton, J. R., \& Robert-McComb, J. J. (2012). Eating disorders in women and children: Prevention, stress management, and treatment (2nd ed.). Boca Raton, FL: Taylor \& Francis Group.

Grabe, S., \& Hyde, J. S. (2006). Ethnicity and body dissatisfaction among women in the United States: A metaanalysis. Psychological Bulletin, 132, 622-640.

Grogan, S. (2008). Body image: Understanding body dissatisfaction in men, women, and children (2nd ed.). New York, NY: Routledge.

Guest, G., Bunce, A., \& Johnson, L. (2006). How many interviews are enough? An experiment with data saturation and variability. Family Health International, 18(1), 59-82. doi: 10.1177/1525822X05279903

Hausenblas, H. A., \& Fallon, E. A. (2006). Exercise and body image: A meta-analysis. Psychology \& Health, 21(1), 33-47. doi: 10.1080/14768320500105270

Henwood, K., \& Pidgeon, N. (2006). Grounded theory. In G. M. Breakwell, S. Hammond, C. Fife-Schaw \& J. A. Smith (Eds.), Research methods in psychology (3rd ed.). London, England: Sage.

Hesse-Biber, S. (1991). Women, weight and eating disorders: A socio-cultural and political-economic analysis. Women's Studies International Forum, 14, 173-191.

Heyes, C. J. (2007). Cosmetic surgery and the televisual makeover. Feminist Media Studies, 7(1), 17-32. doi: $10.1080 / 14680770601103670$

Hill, A. J. (2004). Does dieting make you fat? British Journal of Nutrition, 92, 15-18. doi: http://dx.doi. org/10.1079/BJN20041135

Jackson, J., \& Lyons, A. C. (2012). The perfect body: Men and women negotiate spaces of resistance against beauty and gender ideologies. Women's Studies Journal, 26(1), 25-33.

Jeffreys, S. (2005). Beauty and misogyny: Harmful cultural practices in the west. London, England: Routledge.

Leong, S. L., Madden, C., Gray, A., \& Horwath, C. (2012). Self-determined, autonomous regulation of eating behavior is related to lower body mass index in a nationwide survey of middle-aged women. Journal of the Academy of Nutrition \& Dietetics, 112(9), 1337-1346. doi: http://dx.doi.org/10.1016/j.jand.2012.04.018

Markula, P., Burns, M., \& Riley, S. (2008). Introducing critical bodies: Representations, identities and practices of weight and body management. In S. Riley, M. Burns, H. Frith, S. Wiggins \& P. Markula (Eds.), Critical bodies: Representations, identities and practices of weight and body management. New York, NY: Palgrave Macmillian. 
Matthiasdottir, E., Jonsson, S. J., \& Kristjansson, A. L. (2010). Body weight dissatisfaction in the Icelandic adult population: A normatice discontent? The European Journal of Public Health Access, 1-6. doi: 10.1093/eurpub/ ckq178

McVey, G., Tweed, S., \& Blackmore, E. (2004). Dieting among preadolescent and young adolescent females. Canadian Medical Association Journal, 170(10). doi: 10.1503/cmaj.1031247

Neumark-Sztainer, D., Wall, M., Larson, N. I., Eisenberg, M. E., \& Loth, K. (2011). Dieting and disordered eating behaviors from adolescence to young adulthood: Findings from a 10-year longitudinal study. Journal of the American Dietetic Association, 111(7), 1004-1011. doi: http://dx.doi.org/10.1016/j.jada.2011.04.012

Rodin, J., Silberstein, L. R., \& Striegel-Moore, R. H. (1984). Women and weight: A normative discontent. In R. Sonderegge (Ed.), Nebraska Symposium on Motivation (pp. 267-307). Lincoln, NB: University of Nebraska Press.

Sabik, N. J., Cole, E. R., \& Ward, L. M. (2010). Are all minority women equally buffered from negative body image? Intra-ethnic moderators of the buffering hypothesis. Psychology of Women Quarterly, 34, 139-151.

Strauss, A. (1987). Qualitative analysis for social scientists. New York, NY: Cambridge University Press.

Strauss, A., \& Corbin, J. (1990). Basics of qualitative research: Grounded theory procedures and techniques. Newbury Park, CA: Sage.

Streeter, V. M., Milhausen, R. R., \& Buchholz, A. C. (2012). Body image, body mass index, and body composition. Canadian Journal of Dietetic Practice \& Research, 73(2), 78-83. doi: 10.3148/73.2.2012.78

Tantleff-Dunn, S., Barnes, R. D., \& Larose, J. G. (2011). It's not just a "woman thing:" The current state of normative discontent. Eating Disorders, 19(5), 392-402. doi: 10.1080/10640266.2011.609088

Tiggemann, M. (2004). Body image across the adult life span: Stability and change. Body Image, 1, 29-41. doi: http://dx.doi.org/10.1016/S1740-1445(03)00002-0 